\title{
Unsupervised Image Segmentation with Adaptive Archive-Based Evolutionary Multiobjective Clustering
}

\author{
Chin Wei Bong ${ }^{1}$ and Hong Yoong Lam ${ }^{2}$ \\ 1 School of Computer Science, Universiti Sains Malaysia \\ 2 Department of Cardiothoracic Surgery, Hospital Pulau Pinang \\ Penang, Malaysia \\ bongwendy@gmail.com, drlamhy@yahoo.ca
}

\begin{abstract}
The aim of this paper is to propose and apply state-of-the-art multiobjective scatter search for solving image segmentation problem. The algorithm incorporates the concepts of Pareto dominance, external archiving, diversification and intensification of solutions. The multiobjective optimization method is Archive-based Hybrid Scatter Search (AbYSS) for image segmentation. It utilized fuzzy clustering method with optimization of two fitness functions, viz., the global fuzzy compactness of the clusters and the fuzzy separation. We have tested the methods on two types of grey scale images, namely SAR (synthetic aperture radar) image and CT scan (Computer Tomography) image. We then compared it with fuzzy c-means (FCM) and a popular evolutionary multiobjective evolutionary clustering named NSGA-II. The performance result for the proposed method is compatible with the existing methods.
\end{abstract}

Keywords: Multiobjective clustering, soft computing.

\section{Introduction}

Image segmentation process is defined as the extraction of the important objects from an input image [1]. It partitions the pixels in the image into homogeneous regions, each of which corresponds to some particular information. There are many approaches available for image segmentation including threshold methods [2], morphologic methods [3] and clustering algorithms. However, the segmentation results from these methods are still not satisfactory.

Majority of existing clustering algorithms are based on only one internal evaluation function, which is a single-objective function that measures intrinsic properties of a partitioning such as spatial separation between the clusters or the compactness of the clusters. However, it is sometimes difficult to reflect the quality of partitioning reliably with only one internal evaluation function which may be violated for certain datasets [7]. In this paper, we use multiobjective optimization (MOP) to overcome the defects of the single-objective clustering algorithms, such as FCM. Given that the objective functions (no less than two) for clustering are complementary, the simultaneous optimization of several of 
those objectives may lead to high quality solutions and improve the robustness towards different data properties. MOCK proposed in [9] may be the first application of MOP in data clustering. However, only few applications have been reported in image segmentation [10]. Among those applications, NSGA-II [8] was found to be the most popular method being used so far. It is difficult to apply current MOP clustering technology to image segmentation, owing to an extremely large amount of data need to be handled and thus the handling of population in an evolutionary algorithm (EA) is tedious.

Scatter search [4] is an EA in the sense that it incorporates the concept of population. As compared to other EA, it usually avoids using many random components, and it is based on using a small population, known as the reference set, whose individuals are combined to construct new solutions which are generated systematically. The reference set is initialized from an initial population composed of diverse solutions, and it is updated with the solutions resulting from the local search improvement. Scatter search has been found to be successful in a wide variety of optimization problems [6], and until recently it had been extended to deal with MOPs. Scatter search can act as a powerful local search engine for tasks such as generating missing parts of a Pareto front because of its flexibility and ease of use [11]. Therefore, this paper first proposes and applies the state-of-the-art multiobjective scatter search for image segmentation using Archive-based Hybrid Scatter Search (AbYSS). The framework incorporates the concepts of Pareto dominance, external archiving, diversification and intensification of solution.

In the rest of this paper, we first present our proposal, followed by the experimental conducted to compare the proposed method with other standard image segmentation methods. Finally, we conclude the paper in the last section.

\section{Multiobjective Clustering with AbYSS}

The image segmentation problem is posed as clustering the pixels of the images in the intensity space. Here, a fuzzy clustering algorithm produces a membership matrix $U(X)=\left[\mu_{k j}\right], k=1, \ldots, K$ and $j=1, \ldots, n$, where $\mu_{k j}$ denotes the membership degree of pattern $x_{j}$ to cluster $C_{k}$. Here the individual are made up of real numbers which represent the coordinates of the cluster centers. If individual encodes the centers of $K_{i}$ clusters in $p$ dimensional space then its length $l_{i}$ will be $p \times K_{i}$. In the initial population, each string $i$ encodes the centers of a some $K_{i}$ number of clusters, such that $K_{i}=\left(\operatorname{rand}() \% K^{*}\right)+2$, where $\operatorname{rand}()$ is a function returning a random integer, and $K^{*}$ is a soft estimate of the upper bound of the number of clusters [6]. Therefore, the number of clusters will vary from 2 to $K^{*}+1$. The $K_{i}$ centers encoded in an individual of the initial population are randomly selected distinct points from the input data set.

Two fitness functions, viz., the global fuzzy compactness $J$ of the clusters and the fuzzy separation $S$ are optimized simultaneously[5]. For computing the objective functions, first the centers, $V=\left\{v_{1}, v_{2}, \ldots v_{k}\right\}$ encoded in a given individual are extracted. The fuzzy membership values $\mu_{k j}, i=1, \ldots, K, k=1,2, \ldots, n$ 
are computed using $\mu_{i k}=\sum_{j=1}^{K}{\frac{D\left(v_{i}, x_{k}\right)}{D\left(v_{j}, x_{k}\right)}}^{-2 /(m-1)}, 1 \leq i \leq K ; 1 \leq k \leq n$, where $D\left(v_{i}, x_{k}\right)$ denotes the distance between $i$ th cluster center and $k$ th data point and $m \in[1, \infty]$ is the fuzzy exponent. Each cluster center $v_{j}=1,2, \ldots, K$, is updated using $v_{i}=\frac{\sum_{k=1}^{n} \mu_{i k}^{m} x_{k}}{\sum_{k=1}^{n} \mu_{i k}^{m}}$. The membership values are then recomputed. The variation $\sigma_{i}$ and fuzzy cardinality $n_{i}$ of the $i$ th cluster, $i=1,2, \ldots, K$ are calculated as $\sigma_{i}=\sum_{k=1}^{n} \mu_{i k}^{m} D\left(v_{i}, x_{k}\right), 1 \leq i \leq K$ and $n_{i}=\sum_{k=1}^{n} \mu_{i k}, 1 \leq i \leq K$. The global compactness of the solution represented by the chromosome is then computed as $J=\sum_{i=1}^{K} \frac{\sigma_{i}}{n_{i}}=\sum_{i=1}^{K} \frac{\sum_{k=1}^{n} \mu_{i k}^{m} D\left(z_{i}, x_{k}\right)}{\sum_{k=1}^{n} \mu_{i k}}$.

The other fitness function fuzzy separation $S$ is computed as follows: the center $v_{i}$ of the $i$ th cluster, is assumed to be the center of a fuzzy set . Hence the membership degree of each $v_{j}$ to $v_{i}, j \neq i$, is computed as

$\mu_{i j}=\sum_{l=1, l \neq j}^{K}{\frac{D\left(v_{i}, v_{j}\right)}{D\left(v_{j}, v_{l}\right)}}^{-2 /(m-1)}, i \neq j$.

Subsequently, the fuzzy separation is defined as $S=\sum_{i=1}^{K} \sum_{i=1, j \neq i}^{K} \mu_{i j}^{m} D\left(v_{i}, v_{j}\right)$. In order to obtain compact clusters, the measure $J$ should be minimized. On the contrary, to get well separated clusters, the fuzzy separation $S$ should be maximized. As in this article the multiobjective problem is posed as minimization of both the objectives, hence the objective is to minimize $J$ and $\frac{1}{S}$ simultaneously.

AbYSS is based on the scatter search template adopted from [4]. The template defines six methods, as depicted in Figure 1, are described below:

1) Diversification Generation Procedure: The procedure is the same as that proposed in [4]. The goal is to generate an initial set $\mathrm{P}$ of diverse solutions. This is a simple method based on dividing the range of each variable into a number of subranges of equal size; then, the value for each variable of every solution is generated.

2) Improvement procedure: This procedure is to use a local search algorithm (a simplex method) to improve new solutions obtained from the diversification generation and solution combination methods. The improvement method takes an individual as a parameter, which is repeatedly mutated with the aim of obtaining a better individual. The term "better" is defined here in a similar way to the constrained-dominance approach used in NSGA-II [8].

3) Reference Set Update procedure: The reference set is a collection of both high-quality and diverse solutions that are used to generate new individuals. The set itself is composed of two subsets, RefSet 1 and RefSet2, of size $p$ and $q$, respectively. The first subset contains the best quality solutions in $P$, while the second subset should be filled with solutions promoting diversity.

4) Subset Generation procedure: This procedure generates subsets of individuals, which will be used to create new solutions with the solution combination method. The strategy used considers all pairwise combinations of solutions in the reference set [7]. Furthermore, this method should avoid producing repeated subsets of individuals, i.e., subsets previously generated. 


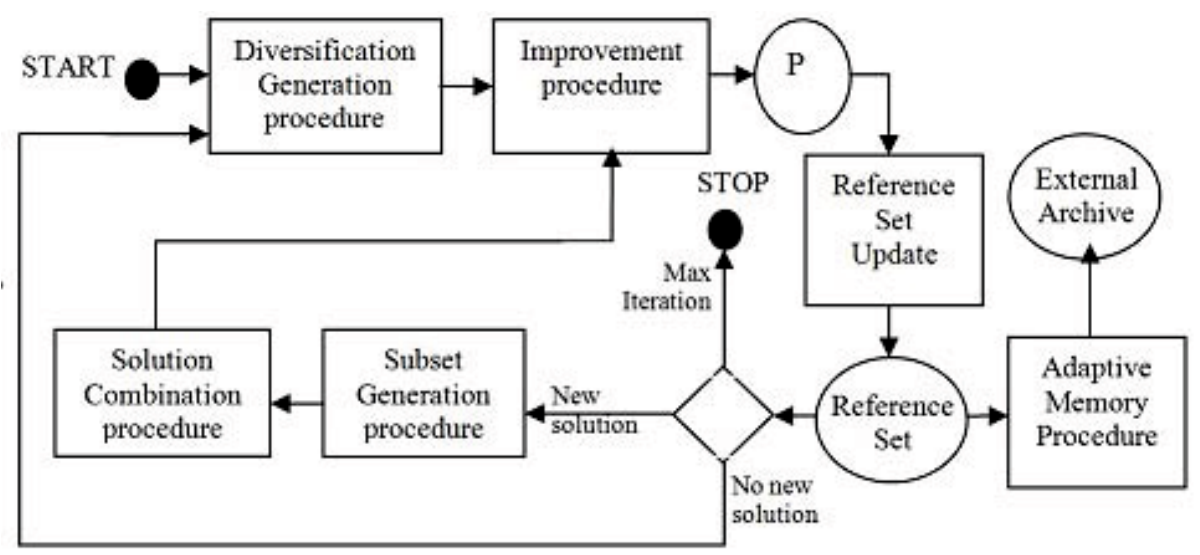

Fig. 1. Template for the six methods in the proposed framework

5) Solution Combination procedure: This procedure is to find linear combinations of reference solutions. The use of a simulated binary crossover operator (SBX) makes AbYSS more robust [6].

6) Adaptive Memory Procedure: The aim of External Archive is to store a record of the non-dominated individuals found during the search in order to keep those individuals producing a well distributed Pareto front. This archive is empty at the beginning of the method. We will continuously update it whenever a new individual or solution is not dominated by the RefSet1. Besides, we use the following rules to reduce the computational requirements: (a) New solution $y$ obtained from $x$ should be used to update External Archive only if it is not dominated by $x$. (b) New potentially Pareto-optimal solutions could be added to External Archive only if they differ enough from all solutions already contained in this set. We propose to use a threshold defining the minimum Euclidean distance in the space of normalized objectives between solutions in External Archive. A new potentially Pareto optimal solution is neglected if it is closer to at least one solution in External Archive than the threshold. We have observed experimentally that the solutions added to External Archive in early iterations have a good chance to be removed from this set in further iterations. In other words the time spent on updating External Archive in early iterations is likely to be lost. Thus, it is possible to neglect updating External Archive in a number of starting iterations.

Initially, the diversification generation method is invoked to generate initial solutions, and each one is passed to the improvement method and the result is the initial set $P$. Then, a fix number of iterations are performed. At each iteration, the reference set is built, the subset generation method is invoked, and the main loop of the scatter search algorithm is executed until the subset generation method stops producing new subsets of solutions. Then, there is a restart phase, which consists of three steps. First, the individuals RefSet 1 in are inserted into 
$P$; second, the best individuals $n$ from the external archive, according to the crowding distance, are also moved to $P$; and, third, the diversification generation and improvement methods are used to produce new solutions for filling up the set $P$. The idea of moving $n$ individuals from the archive to the initial set is to promote the intensification capabilities of the search towards the Pareto front already found. The intensification degree can vary depending on the number of chosen individuals. The best single solution is then selected based on the method introduced by MetaClustering algorithm (MCLA)[5].

\section{Experimental Study}

We have compared our algorithm with standard FCM and a popular evolutionary multobjective clustering, NSGA-II. For NSGA-II, we executed 100 generations with fixed population size 50 . The crossover and mutation probabilities are fixed as 0.8 and 0.01 , respectively. FCM is executed for a maximum of 100 iterations, with $\mathrm{m}$, the fuzzy exponent, equal to 2.0. First, the experiment was done for a 3class SAR images obtained from http://www.sandia.gov/radar/imageryku.html. The image is a $\mathrm{Ku}$-band SAR image with one meter spatial resolution in the area of Rio Grande River near Albuquerque, New Mexico, USA. This image consists of three types of land cover, namely, the river, the vegetation and the crop. Then, the experiment was extended to real CT scan image for Lung obtained from Penang Hospital, Malaysia. The results of the algorithms are compared with two cluster validity functions: partition coefficient, $V_{p c}(U)=\frac{\sum_{j=1}^{n} \sum_{i=1}^{c} \mu_{i j}^{2}}{n}$ and partition entropy value, $V_{p e}(U)=\frac{1}{n} \sum_{j=1}^{n} \sum_{i=1}^{c} \mu_{i j} \log \mu_{i j}$ [7]. The clustering result is better with a higher $V_{p c}(U)$ value and a lower $V_{p e}(U)$ value. Table 1 shows the performance comparison of FCM, NSGA-II and the proposed method in terms of cluster validity functions with both index. The values of $V_{p c}(U)$ are higher in the proposed method than the FCM and NSGA-II methods. Meanwhile, the values of $V_{p e}(U)$ are lower in the proposed method than the FCM and NSGA-II methods. This result indicates that the proposed method is outperformed than other two methods. Fig. 2 shows the example of images and their segmented results. For SAR image, FCM and the proposed method are better than NSGA-II in terms of the regional consistency of the water and the buildings region. However, all the three algorithms cannot achieve the visually correct segmentation in the right top region, i.e. FCM, and the proposed method misclassifies a big area of land region as the water. Comparatively, the proposed method performs best in this area where the misclassification is not so serious as FCM and NSGA-II.

Table 1. Result of partition coefficient and partition entropy for the two types of images with different methods

\begin{tabular}{|c|c|c|c|c|c|c|c|c|}
\hline Methods & Index & FCM & NSGA-II & Proposed & Index & FCM & NSGA-II & Proposed \\
\hline SAR IMAGE & $V_{p e}$ & 0.14230 & 0.14025 & 0.11227 & $V_{p c}$ & 0.91031 & 0.91106 & 0.92180 \\
\hline CT Scan IMAGE & $V_{p e}$ & 0.06910 & 0.05150 & 0.0403 & $V_{p c}$ & 0.93113 & 0.94170 & 0.95943 \\
\hline
\end{tabular}




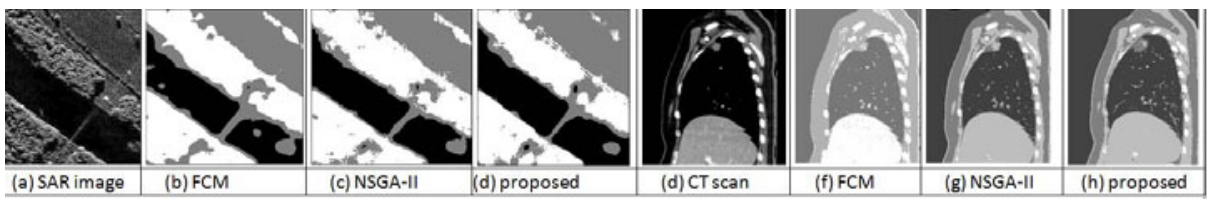

Fig. 2. Two 3-Class SAR images and their segmentation results

\section{Conclusion}

Most real-world image segmentation problems involve simultaneously optimizing multiple criteria where considerations of trade-offs is important. This paper has presented the concepts of Pareto dominance, external archiving, diversification and intensification of solutions. The performance of the proposed method was encouraging and future work will focus on enhancing the performance speed of the algorithm. More in-depth research will be conducted in analysing the variables used in the algorithm. Besides, we are also interested to include a few more criteria into our existing bi-criteria optimization.

\section{References}

[1] Gonzalez, R.C., Woods, R.E.: Digital Image processing. Prentice-Hall, Englewood Cliffs (2007)

[2] Zaart, A.E., Ziou, D., Wang, S., Jiang, Q.: Segmentation of SAR images using mixture of gamma distribution. Pattern Recognition 35(3), 713-724 (2002)

[3] Lemarechal, C., Fjortoft, R., Marthon, P., Cubero-castan, E., Lopes, A.: SAR image segmentation by morphological methods. In: Proc. SPIE, vol. 3497, pp. 111-121 (1998)

[4] Glover, F., Laguna, M., Martí, R.: Scatter search. In: Ghosh, A., Tsutsui, S. (eds.) Advances in Evolutionary Computing: Theory and Applications, pp. 519537. Springer, Heidelberg (2003)

[5] Mukhopadhyaya, A., Maulik, U.: A multiobjective approach to MR brain image segmentation. Applied Soft Computing 11, 872-880 (2011)

[6] Nebro, A.J., Luna, F., Alba, E., Dorronsoro, B., Durillo, J.J., Beham, A.: AbYSS: Adapting Scatter Search to Multiobjective Optimization. IEEE Transactions on Evo. Comp. 12(4) (2008)

[7] Bezdek, J.C.: Cluster validity with fuzzy sets. Cybernetics and Systems, 58-73 (1974)

[8] Deb, K., Pratap, A., Agarwal, S., Meyarivan, T.: A fast and elitist multiobjective genetic algorithm: NSGA-II. IEEE Trans. on Evolutionary Computation 6(2), 182-197 (2002)

[9] Handl, J., Knowles, J.: An evolutionary approach to multiobjective clustering. IEEE Trans. on Evolutionary Computing 11(1), 56-76 (2007)

[10] Saha, S., Bandyopadhyay, S.: Unsupervised pixel classification in satellite imagery using a new multiobjective symmetry based clustering approach. In: TENCON IEEE Region 10 Conference (2008)

[11] Coello, C.A.C.: Evolutionary multi-objective optimization: some current research trends and topics that remain to be explored. Frontiers of Computer Science in China 3(1), 18-30 (2009) 